\title{
First Results from the HDMS experiment in the Final Setup
}

\author{
H.V. Klapdor-Kleingrothaus ${ }^{*}$, A. Dietz, G. Heusser, \\ I.V. Krivosheina, D. Mazza, H. Strecker, C. Tomei \\ * Spokesman of the Collaboration
}

\begin{abstract}
The Heidelberg Dark Matter Search (HDMS) is an experiment designed for the search for WIMP dark matter. It is using a special configuration of Ge detectors, to efficiently reduce the background in the low-energy region below $100 \mathrm{keV}$. After one year of running the HDMS detector prototype in the Gran Sasso Underground Laboratory, the inner crystal of the detector has been replaced with a HPGe crystal of enriched ${ }^{73} \mathrm{Ge}$. The final setup started data taking in Gran Sasso in August 2000. The performance and the first results of the measurement with the final setup are discussed.
\end{abstract}

\section{Introduction}

Weakly Interacting Massive Particles (WIMPs) are leading candidates for the dominant form of matter in our Galaxy. These relic particles from an early phase of the Universe arise independently from cosmological considerations in supersymmetric particle physics theories as neutralinos - the lightest supersymmetric particles.

Direct WIMP detection experiments exploit the elastic WIMP scattering off nuclei in a terrestrial detector [1]. However, detecting WIMPs is not a simple task. Their interaction with matter is very feeble $\left(\sigma \leq \sigma_{\text {weak }}\right)$ and predicted rates in supersymmetric models range from 10 to $10^{-5}$ events per kilogram detector material and day [2, 3, , , 5, 6]. Moreover, for WIMP masses between a few $\mathrm{GeV}$ and $1 \mathrm{TeV}$, the energy deposited by the recoil nucleus is less then $100 \mathrm{keV}$. Thus, in order to be able to detect a WIMP, an experiment with a low-energy threshold and an extremely low radioactive background is required. Since the reward would be no less than discovering the dark matter in the Universe, a huge effort is put into direct detection experiments. More than 20 experiments are running at present and even more are planned for the future (for recent reviews see [7, 8, 9]). 


\section{Description of the experiment}

In direct detection experiments looking for WIMPs one of the main goals is the reduction of background events since the sensitivity of experiments roughly scales with the obtained background level.

HDMS operates two ionization HPGe detectors in a unique configuration [10]. A small, p-type Ge crystal is surrounded by a well-type Ge crystal, both being mounted into a common cryostat system (see Figure 1 for a schematic view). Two effects are expected to reduce the background of the inner target detector with respect to our best measurements with the Heidelberg-Moscow experiment [11]. First, the anticoincidence between the two detectors acts as an effective suppression of multiple scattered photons. Second, we know that the main radioactive background of Ge detectors comes from materials situated in the immediate vicinity of the crystals. In the case of HDMS the inner detector is surrounded (apart from the thin isolation) by a second Ge crystal - one of the radio-purest known materials. In order to reduce the background with respect to the prototype detector, the following changes has been made:

- The inner detector has been replaced by a crystal grown out of HPGe material enriched in ${ }^{73} \mathrm{Ge}$. This has the effect that the mother isotope of cosmogenic ${ }^{68} \mathrm{Ge}$ production, ${ }^{70} \mathrm{Ge}\left({ }^{70} \mathrm{Ge}(\mathrm{n}, \mathrm{t}){ }^{68} \mathrm{Ge}\right)$, is deenriched by up to a factor of 50 . Thus the decay of ${ }^{68} \mathrm{Ge}$ will be suppressed by this factor with respect to a natural HPGe crystal. The usage of enriched ${ }^{73} \mathrm{Ge}$ also allows the evaluation for spin dependent WIMP nucleon crosssections.

- The contacts of the HPGe crystals were pinched in order to avoid the use of soldering tin inside the detector cap.

- The crystal holder system has been replaced. The new material is from the same

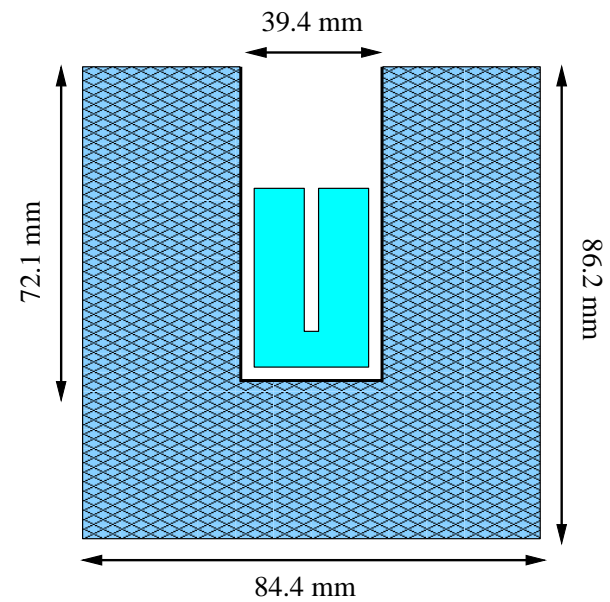

Figure 1: Schematic view of the HDMS detector configuration. All events which are seen in both the inner and the outer Ge-detector can be considered as background events, not resulting from a WIMP-nucleon recoil. 
Table 1:

Technical data of the detector in the final setup.

\begin{tabular}{lrr}
\hline Property & Inner Detector & Outer Detector \\
\hline \hline Crystal Type & $\mathrm{p}$-type & $\mathrm{n}$-type \\
Mass [g] & 202 & 2111 \\
Active Volume $\left[\mathrm{cm}^{3}\right]$ & 37 & 383 \\
Crystal diameter [mm] & 35.2 & 84.4 \\
Crystal length [mm] & 40.3 & 86.2 \\
Operation Bias [V] & +2500 & -1500 \\
Energy resolution FWHM $(1332 \mathrm{keV})[\mathrm{keV}]$ & 1.87 & 4.45 \\
Energy threshold [keV] & 4.0 & 7.5 \\
\hline
\end{tabular}
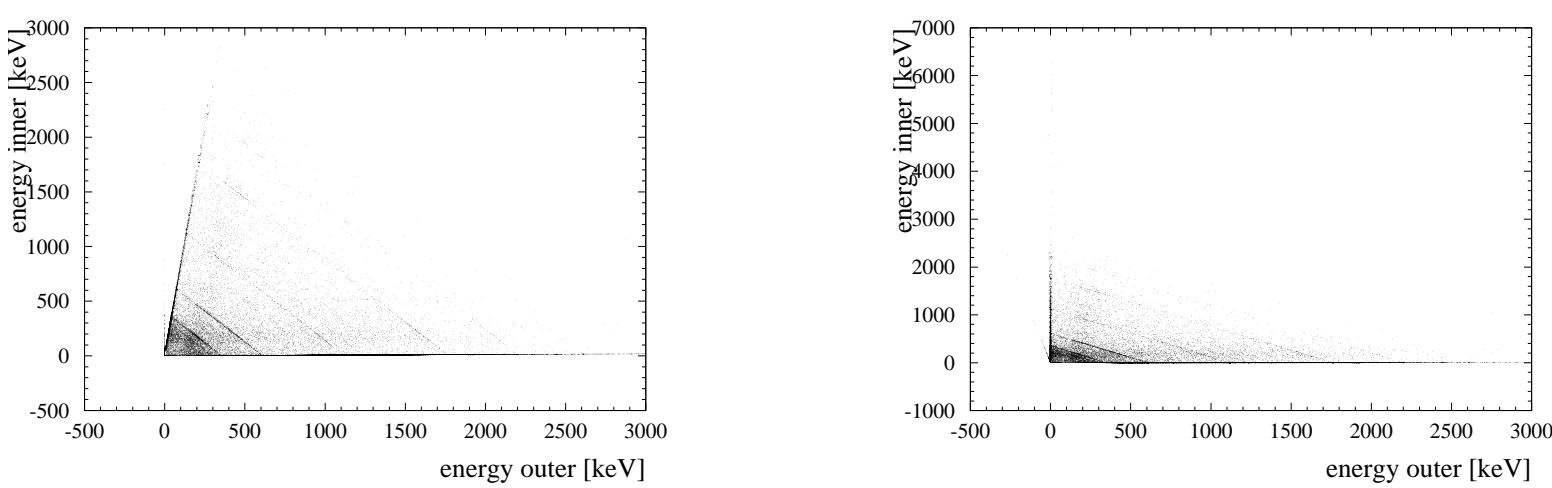

Figure 2: Scatter plot of the measured data. Each dot corresponds to one event. The y- and $\mathrm{x}$-axis display the energy deposited in the inner- and outer detector, respectively. Left: Before the correction for pick-up signals the zero energy axes have a non-zero slope. Right: After the correction the zero energy axes correspond to the $\mathrm{y}$ - and $\mathrm{x}$ - axes.

sample as the material used in the Heidelberg-Moscow $\beta \beta$ experiment, which is known to be very clean.

Some technical details are listed in Table 1 .

\subsection{The anti-coincidence and the crosstalk}

Due to the special concentric design, the spatial separation between the two detectors is very small. This gives rise to pick-up signals. If one of the detectors sees an event, a cross talk signal is induced in the other one.

Let $E_{i}$ and $E_{o}$ be the real deposited energies in the inner and the outer detector, respectively. The cross talk effect causes pickup energies so that the measured energies are:

$$
E_{i}^{\prime}=E_{i}+k_{i o} \times E_{o} \quad \text { and } \quad E_{o}^{\prime}=E_{o}+k_{o i} \times E_{i}
$$




\begin{tabular}{lcc}
\hline Property & $\begin{array}{c}\text { Inner Detector } \\
\text { final setup }\end{array}$ \\
\hline \hline Threshold & $(4.0 \pm 0.2) \mathrm{keV}$ & $(7.5 \pm 0.2) \mathrm{keV}$ \\
\hline $\begin{array}{l}\text { Energy res. } \\
\text { at zero keV } \\
\text { extrapolated }\end{array}$ & $(0.76 \pm 0.05) \mathrm{keV}$ & $(2.82 \pm 0.06) \mathrm{keV}$ \\
\hline $\begin{array}{l}\text { at zero keV } \\
\text { after correction }\end{array}$ & $(0.83 \pm 0.01) \mathrm{keV}$ & $(2.91 \pm 0.04) \mathrm{keV}$ \\
\hline at $81 \mathrm{keV}$ & $(0.95 \pm 0.03) \mathrm{keV}$ & \\
\hline at $344 \mathrm{keV}$ & & $(3.03 \pm 0.03) \mathrm{keV}$ \\
\hline at $1408 \mathrm{keV}$ & & $(4.46 \pm 0.02) \mathrm{keV}$ \\
\hline
\end{tabular}

Table 2: Energy resolutions for different energies and thresholds of the current final detector configuration.

with the slopes $k_{i o}$ and $k_{o i}$. The true energies deposited inside the detectors is given by the relation

$$
E_{i}=\frac{E_{i}^{\prime}-k_{i o} \times E_{o}^{\prime}}{1-k_{i o} \times k_{o i}} \quad \text { and } \quad E_{o}=\frac{E_{o}^{\prime}-k_{o i} \times E_{i}^{\prime}}{1-k_{i o} \times k_{o i}}
$$

Recording spectra of calibration sources with the list mode allows to visualize this pickup signal (see Fig. 2). The anti-coincidence cut between the two detectors to recognize multiple scattered background events can only be applied, if the cross talk is eliminated. This cut is made by defining all events as background events, in which an energy deposition is seen in both detectors above the energy threshold of the proper detector.

It was shown that the cross talk is linear with energy and stable over time and can be corrected for off-line [13]. Once the correction is made, the anti-coincidence can be applied.

\subsection{Measurements at the Gran Sasso underground laboratory}

The final setup of the HDMS was installed at the LNGS in August 2000, the data used for the analysis are taken from February 2001 to September 2001 in order to let the cosmogenic isotopes decay. The time stability of the energy resolution, threshold and calibration parameters (slope and intercept of energy calibration) is checked by weekly measurements with a ${ }^{152} \mathrm{Ba}^{228}$ Th-source. This have been discussed elsewhere [12, 14]. The measured energy resolutions and thresholds of the detectors are listed in Tab. 2 . They correspond to standard values for detectors of this size.

The individual typical duration of a run was about 23 hours. The experiment was stopped daily and the most important detector parameters like leakage current and mean count rates were checked. No substantial fluctuations were recorded.

After the individual runs were calibrated and corrected for the crosstalk, they were added to provide the sum spectra. From the sum spectrum of the inner detector (after the anti-coincidence cut) the limits on WIMP dark matter can be extracted. 

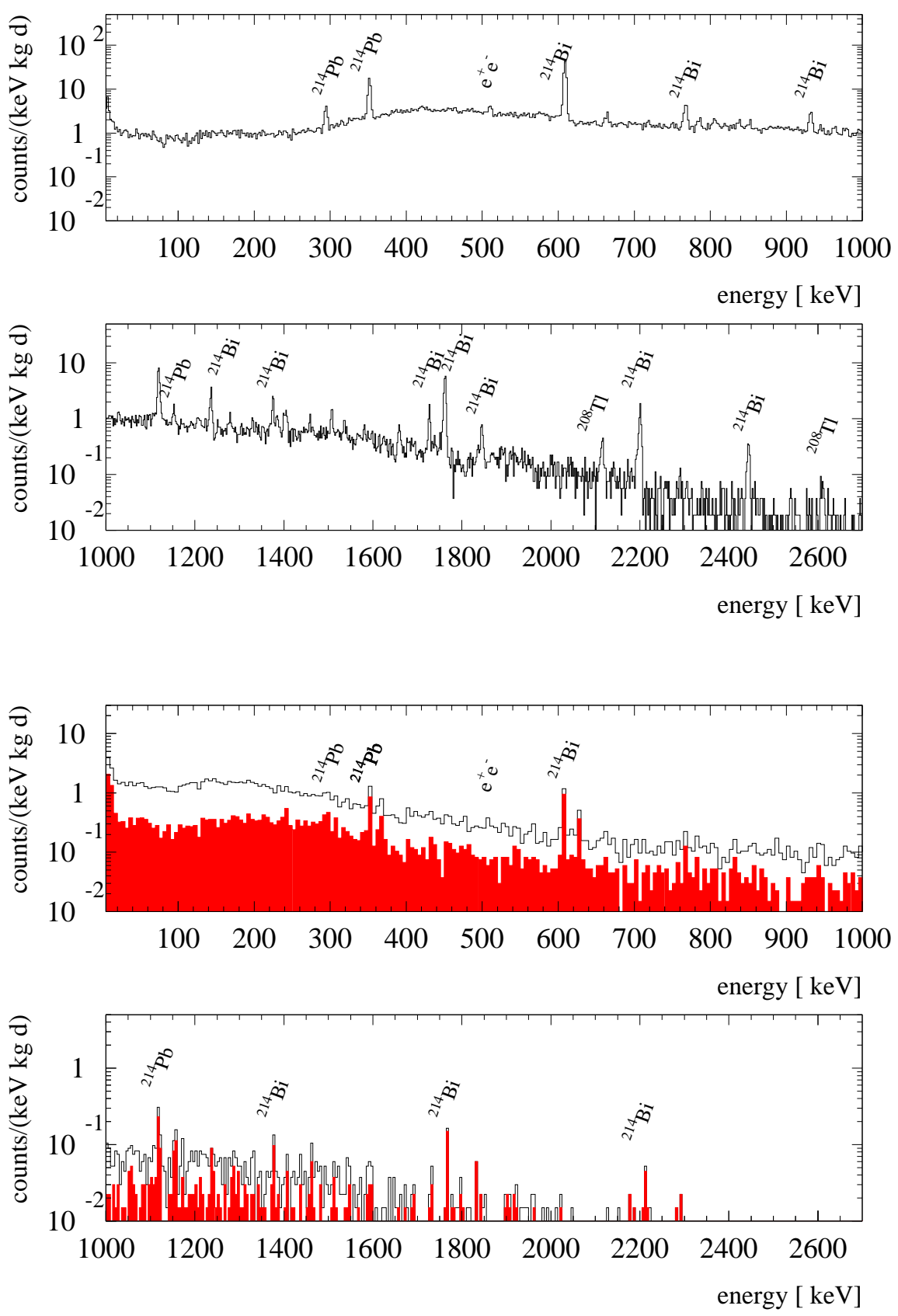

Figure 3: Spectra of the HDMS detectors of the final setup after a total measuring time of 132.4 days. Upper figure: outer detector; lower figure: inner detector; The open histogram denotes the overall spectrum, the filled histogram corresponds to the spectrum after the anti-coincidence cut with the outer detector. The most prominent lines are labeled.

Although the statistics of the inner detector is low, the sum-spectrum (see Fig. 3) show some lines of isotopes from the U/Th natural decay chains as well as some X-ray lines which are dominating the region below $10 \mathrm{keV}$ (see Fig. 团). The most obvious structure 


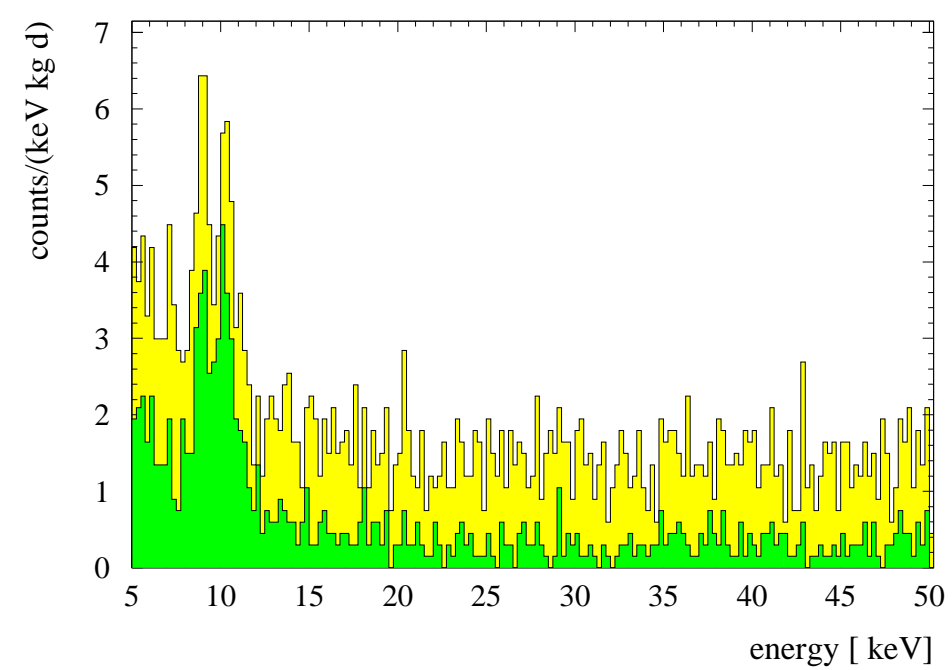

Figure 4: Low-energy spectrum of the inner, enriched Ge detector before and after (open and solid histograms, respectively) the anti coincidence is applied with the outer Ge detector. The internal, low-energy $\mathrm{x}$ rays are not removed by the anti-coincidence.

in the low energy region is a peak at $10.37 \mathrm{keV}$ resulting from the decay of ${ }^{68} \mathrm{Ge}$ and a peak around $9 \mathrm{keV}$, which could arise from ${ }^{65} \mathrm{Zn}$. Note that for the low energy region (Fig. 4) there are no more indications for a contamination with ${ }^{210} \mathrm{~Pb}$ in comparison with the results from the prototype-detector [12]. Also the structure at $32.5 \mathrm{keV}$, which is meanwhile understood (see [15]), vanished completely.

If the anti-coincidence is evaluated in the energy region between $50 \mathrm{keV}$ and $100 \mathrm{keV}$, the background reduction factor is 4.5. The counting rate after the anti-coincidence in this energy region is 0.27 events $/(\mathrm{kg} \mathrm{d} \mathrm{keV}$ ) (from February 2001), thus comparable to the value measured in the Heidelberg-Moscow experiment with the enriched detector ANG2 [11. In the energy region between $11 \mathrm{keV}$ and $40 \mathrm{keV}$ the background index is 0.43 events $/(\mathrm{kg} \mathrm{d} \mathrm{keV.)}$.

\subsection{Dark Matter limits}

Since many cosmogenic isotopes have half-lifes below 300 days, typically the count rate in low-level detectors decreases considerably after one year of storage underground.

For this reason only the last 132.4 days, corresponding to $26.74 \mathrm{~kg}$ d of measurement for the inner detector were used for the evaluation of the final HDMS setup data. The procedure of extracting limits on WIMP dark matter from the obtained spectrum follows the method described in [1].

The resulting upper limit is shown in the exclusion plot in figure 5. Already now the limit given by the HDMS experiment is slightly better in the low energy range than the limit given by the Heidelberg-Moscow $\beta \beta$ experiment for low WIMP masses. This due to the fact that the energy threshold of $5 \mathrm{keV}$ has been obtained for this measurement (compare to $9 \mathrm{keV}$ threshold of the Heidelberg-Moscow $\beta \beta$ experiment [11]). 


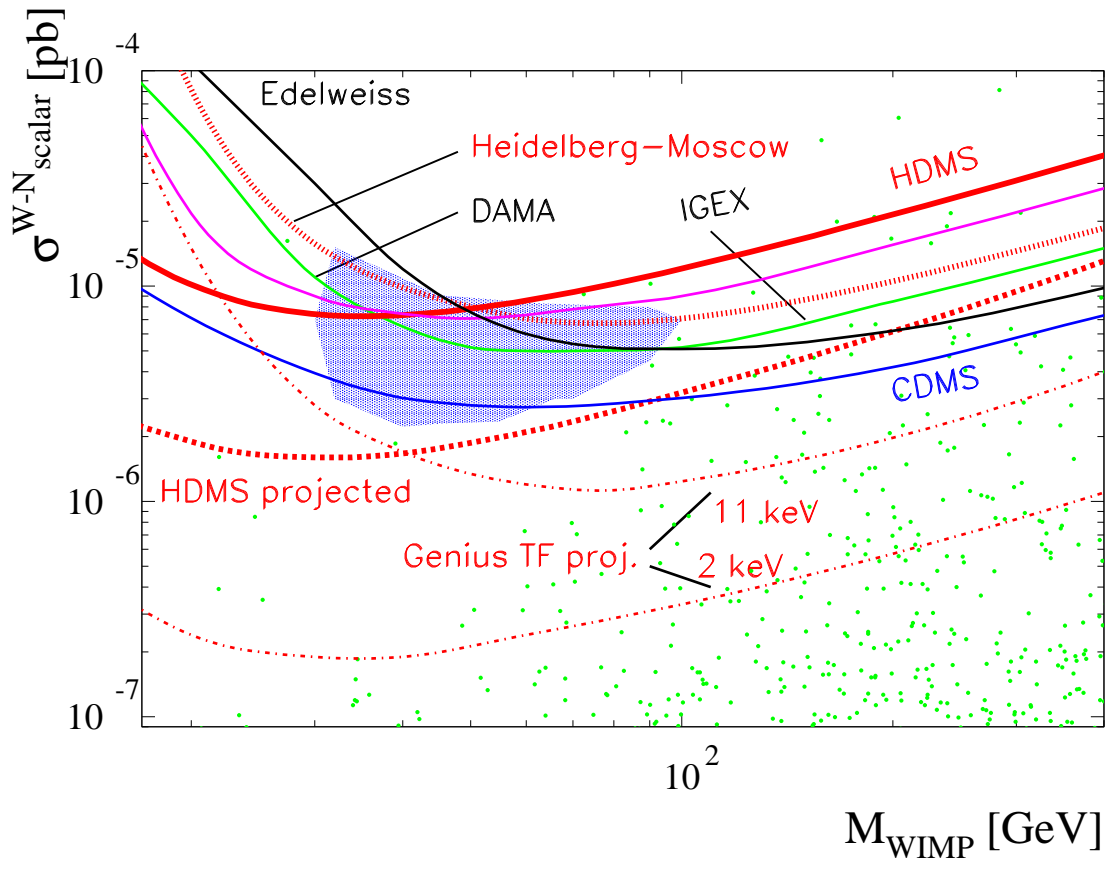

Figure 5: Exclusion plot for the presently most sensitive WIMP dark matter direct search experiments. The shaded area represents the $3 \sigma$ region allowed by the DAMA experiment 16]. Already now, some parts of this region can be tested by the HDMS experiment. Also shown are the present limits from the Heidelberg-Moscow experiment [11, the DAMA experiment [17, the CDMS experiment [18], the IGEX experiment [19], the Edelweiss experiment [20] and future expectations for HDMS and GENIUS-TF.

\section{Conclusion}

The detectors for the final HDMS setup, constructed with some improvements made to reduce the background and including an inner detector from enriched ${ }^{73} \mathrm{Ge}$, were installed in the LNGS in August 2000. It took data for 132.4 days corresponding to $26.74 \mathrm{~kg}$ days. The offline correction for the crosstalk has been applied to the data and a sum spectrum was created, where most of the background sources were identified. The background reduction factor in the inner detector through anti-coincidence is about 4.5. The background in the low-energy region of the inner detector (with exception of the region still dominated by cosmogenic activities) is already comparable to the most sensitive dark matter search experiments. It will be further decreasing due to the decay of cosmogenic isotopes, and with an improved smaller threshold HDMS should be able to test the DAMA evidence region within about three years.

\section{References}

[1] M. W. Goodman and E. Witten, Phys. Rev. D 31 (1985) 3059.

[2] G. Jungmann, M. Kamionkowski, K. Griest, Phys. Rep. 267 (1996) 195 
[3] V. Bednyakov, H.V. Klapdor-Kleingrothaus, S.G. Kovalenko, Y. Ramachers, Z. Phys. A 357 (1997) 339

[4] Bottino et al., hep-ph/0001309.

[5] V. Bednyakov, H.V. Klapdor-Kleingrothaus, Phys. Rev D 62 (2000) 043524 and hep-ph/9908427

[6] V. Bednyakov, H.V. Klapdor-Kleingrothaus, hep-ph/0011233

[7] H.V. Klapdor-Kleingrothaus and Y. Ramachers, Euro. Phys. J. A 3 (1998) 85

[8] L. Baudis and H.V. Klapdor-Kleingrothausin Proc. of Beyond the Desert 1999, ed. by H.V. Klapdor-Kleingrothaus, I.V. Krivosheina (IOP Bristol 2000), p.881

[9] Y. Ramachers, astro-ph/9911260 and in Proc. XI Rencontres de Blois, Frontiers of Matter, France, June 27 - July 3, 1999

[10] L. Baudis, J. Hellmig, H.V. Klapdor-Kleingrothaus, B. Majorovits, Y. Ramachers, H. Strecker, Internal Report, Proposal MPI H-V2-1998 and Nucl. Inst. Meth. A 385 (1997) 265

[11] HEIDELBERG MOSCOW COLLABORATION, Phys. Rev. D 59 (1999) 022001 and Preprint hep-ex/9811045

[12] L. Baudis, A. Dietz, B. Majorovits, F. Schwamm, H. Strecker, H.V. KlapdorKleingrothaus, Phys. Rev. D 63 (2000) 022001, astro-ph/0008339

[13] Y.A. Ramachers, PhD thesis, University of Heidelberg, 1998

[14] L. Baudis, PhD thesis, University of Heidelberg, 1999

[15] H.V. Klapdor-Kleingrothaus in Proc. of the Third Int. Conference on Dark Matter in Astro- and Particle Physics (DARK 2000), Heidelberg, Germany, 10-14 July 2000, edited by H.V. Klapdor-Kleingrothaus and B. Majorovits, Springer, Heidelberg 2000

[16] R. Bernabei et. al., Phys Lett B 480 (2000) 23

[17] R. Bernabei et. al., Phys Lett B 389 (1996) 757

[18] R. Abusaidi et. al, Phys. Rev. Lett 84 (2000) 5699

[19] A. Morales, hep-ex/0110061

[20] A. Benoit et. al, astro-ph/0106094 